\title{
La Gerencia de proyectos estratégicos en la industria farmacéutica en Puerto Rico
}

\author{
Francisco Quiles Torres \\ Universidad de Puerto Rico, Río Piedras
}

Recibido: 21 de febrero de 2014

Aceptado: 8 de mayo de 2014

\author{
Brunilda Aponte Torres \\ Universidad del Turabo, Puerto Rico
}

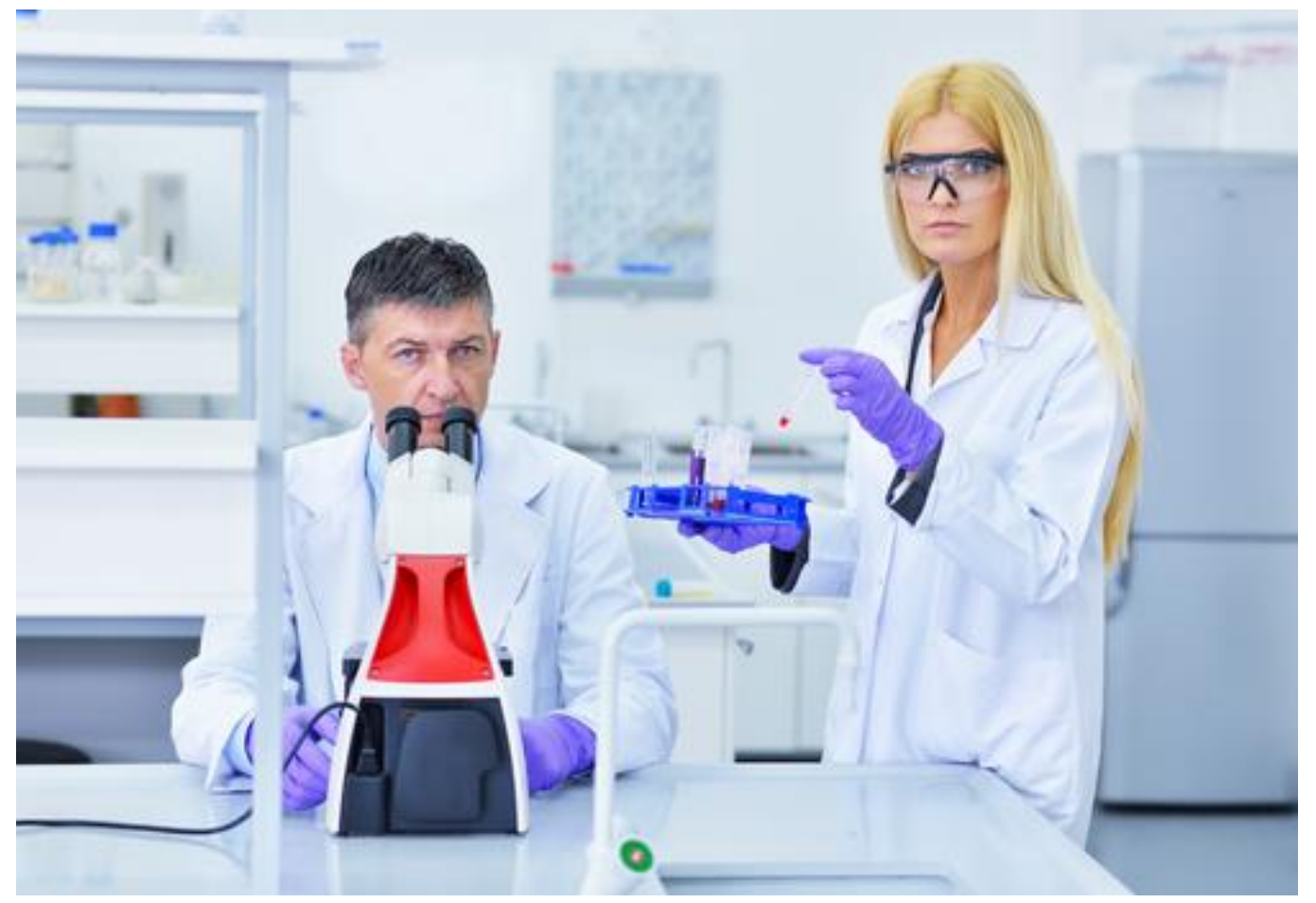

Imágenes utilizadas bajo licencia de uso por Shutterstock.com

\begin{abstract}
Due to the limited time protection that a patent provides a new pharmaceutical product in the market, organizations need to be agile in launching them to maximize their return on investment and need qualified project managers to accomplish it. Competency models have been developed in the United States, which describes the required soft and hard skills that a manager must have in order to be successful in the execution of general as well as strategic projects, but is unknown if they would apply to the management of projects at the pharmaceutical industry in Puerto Rico. This research presents the competency model develop by Rodríguez (2005) and evaluates its applicability in determining the competences needed to manage projects in the pharmaceutical industry sector in Puerto Rico. Results demonstrate that the model has applicability in Puerto Rico, but the relative importance of every competency varied versus the results on the previous study, which indicates that the importance of the competencies may vary depending on the environment.
\end{abstract}

KEY WORDS: project management, strategy, pharmaceutical industry, project manager, competency 



\section{RESUMEN}

Dada la limitada protección de tiempo que una patente le provee a un producto farmacéutico en el mercado, las empresas deben de ser ágiles en lanzarlos al mercado para maximizar sus ganancias. Esto se viabiliza a través de proyectos estratégicos guiados por personal altamente cualificado. Existen en los Estados Unidos modelos que identifican las destrezas de importancia en el manejo de proyectos estratégicos, pero se desconoce si poseen aplicabilidad en Puerto Rico. Se realiza una investigación utilizando el modelo de destrezas técnicas y gerenciales desarrollado por Rodríguez (2005) y se evalúa su aplicabilidad en la industria farmacéutica en Puerto Rico a través de un cuestionario dirigido a gerentes de proyectos. Los resultados comprueban que el modelo de destrezas identificadas por Rodríguez (2005) tiene aplicabilidad en la industria farmacéutica en Puerto Rico, pero con variantes en el nivel de importancia de cada una de ellas, lo cual indica que la importancia de las destrezas depende del entorno estudiado.

PALABRAS CLAVES: gerencia de proyectos, estrategia, industria farmacéutica, gerente de proyectos, destrezas.

\section{Introducción}

a economía de Puerto Rico ha experimentado cambios estructurales importantes en las últimas décadas, en las que el empleo manufacturero se ha reducido mientras que el empleo en las industrias de servicio ha aumentado. La globalización ha traído mayor competencia de Puerto Rico con otros países para atraer empresas extranjeras y así aumentar su nivel de empleo. Una de las industrias que ha sufrido cambios sustanciales en su estructura es la farmacéutica, la cual se encuentra establecida en Puerto Rico desde el comienzo de la década del 1960, y es desde entonces uno de los sectores industriales más importante de Puerto Rico. Para el año 2002, 16 de los 20 medicamentos por prescripción más recetados en el mundo se manufacturaban en Puerto Rico y este sector sustentaba a 29,200 empleos directos (PRIDCO, 2006). Sin embargo, la eliminación de la sección 936 del Código de Rentas Internas Federal "Internal Revenue Code" (IRC), realizado por el Congreso de los Estados Unidos para finales de la década del 1990, trastocó el impulso económico de dicho sector, el cual perdió gran parte de sus exenciones económicas que lo hacían superior a inversiones en otras partes de los Estados Unidos. Dichos cambios han requerido que el sector farmacéutico en Puerto Rico se reinvente, buscando una mayor eficiencia en sus operaciones y una reducción en sus costos, para así poder competir con los ofrecimientos de localidades en los Estados Unidos y otros países, tales como Irlanda y Singapur.

Para lograr una mayor empleabilidad de los recursos humanos y enfrentar con éxito la competencia internacional, Puerto Rico debe disponer de trabajadores con las destrezas requeridas para llenar los puestos disponibles en el Siglo XXI. Un estudio realizado en Puerto Rico en el 2005 identificó las destrezas básicas que deben poseer los empleados en términos generales y de los resultados se pueden destacar dos factores relevantes a esta investigación: (1) se deben establecer maneras efectivas para una mejor comunicación entre los sectores académicos y las empresas, de manera que las destrezas de los graduados de las escuelas superiores y vocacionales, así como de los egresados de programas a nivel universitario, respondan más efectivamente al mundo del trabajo; y (2) se deben continuar realizando estudios similares dirigidos a industrias específicas, para lograr tener mayor información sobre las destrezas requeridas en dichas industrias y poder canalizarlas a las instituciones educativas de la Isla. (Enchautegui, Rodríguez y Segarra, 2005). 
Gerencia de Proyectos en la Industria Farmacéutica

Dado que la patente de un nuevo producto tiene una duración de alrededor de 15 años, las empresas necesitan agilizar la entrada del producto al mercado, buscando ser los primeros para así poder maximizar sus ganancias durante el periodo de no competencia que les brindan las patentes. Las empresas desarrollan sus planes estratégicos de lanzamiento de nuevos productos a través de proyectos, en los que se utiliza el capital financiero y humano (personal) de la empresa para lograr las metas previamente establecidas (Cleland y King, 1983). Las empresas farmacéuticas en general requieren personal altamente cualificado en el área de gerencia de proyectos, con los conocimientos y las destrezas apropiadas para poder ejecutar los proyectos de acuerdo al plan trazado (Alameda, 2006).

Existe un sin número de definiciones para los términos proyecto y gerencia de proyectos en la literatura. El Instituto de Gerencia de Proyectos ("Project Management Institute", conocido por PMI, por sus siglas en inglés) define un proyecto como una gestión temporal que se realiza para crear un producto, servicio o resultado único, y a la gerencia de proyectos como la aplicación del conocimiento, destrezas, herramientas y técnicas en las actividades del proyecto para lograr los requerimientos del mismo. Todo proyecto establece unas metas, la cuales están enmarcadas en tres variables fundamentales: costo, tiempo y alcance del trabajo. Estos tres factores se encuentran muy relacionados entre sí y un cambio en una de estas variables ocasiona cambios en las demás. La relación de estas variables de forma gráfica se presenta en la Figura 1.



Figura 1. Relación de variables costo, tiempo y alcance de trabajo en un proyecto (Augsburg College 2007).

Los conocimientos que necesitan los gerentes de proyectos y su grupo de trabajo para manejar un proyecto varían dependiendo de la naturaleza del proyecto, mas sin embargo el modelo presentado por PMI en su "Project Management Book of Knowledge" (PMBOK, 2004), ilustra los elementos básicos que deben existir. PMI ofrece una certificación en proyectos, denominada "Project Management Professional" (PMP), a través de la cual adiestran al personal en los principios del PMBOK. El modelo del PMBOK se muestra en la Figura 2.

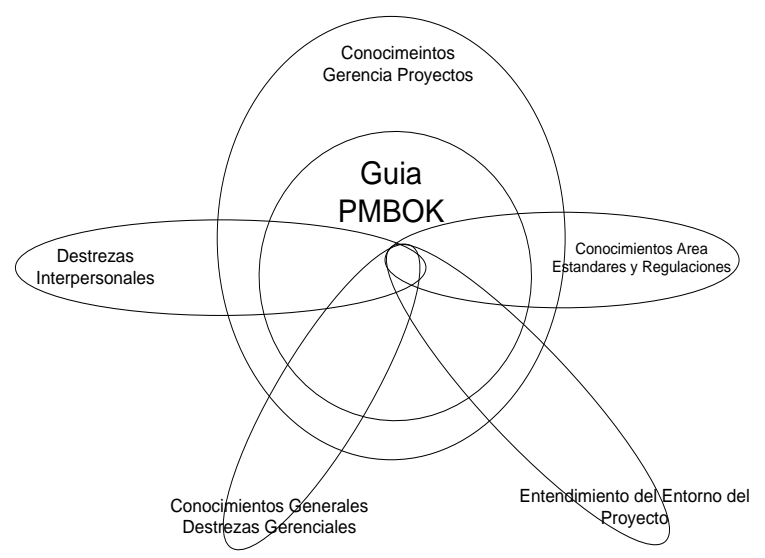

Figura 2. Áreas de Experiencia Necesarias para el Grupo de Proyecto, (PMI, 2003).

Es importante señalar que no todos los miembros del equipo de trabajo en un proyecto tienen que necesariamente ser 
diestros en todas las disciplinas para que el proyecto sea exitoso, sino que en realidad se necesita que todos los conocimientos y las competencias estén cubiertos con los conocimientos y las habilidades combinadas de los miembros. Basado en los retos que enfrenta la industria en Puerto Rico y en la complejidad que envuelve el manejo de proyectos de índole estratégicos, surge la necesidad de conocer cuáles son las competencias o destrezas que debe poseer un gerente de proyectos para el manejo efectivo de los mismos. Una búsqueda de literatura realizada no arrojó ningún modelo que describa las destrezas que son necesarias para el manejo de proyectos en la industria farmacéutica en Puerto Rico.

Investigación: Planteamiento del Problema, Objetivos, Metodología y Variables

El objetivo primordial de la investigación fue determinar cuáles son las destrezas que debe poseer un gerente de proyectos para manejar adecuadamente proyectos estratégicos en la industria farmacéutica de Puerto Rico. Los objetivos específicos fueron: determinar las destrezas técnicas y gerenciales de mayor importancia en el reclutamiento de gerentes de proyecto estratégicos para la industria farmacéutica en Puerto Rico, desarrollar el perfil de los gerentes de proyecto en esta industria, determinar si poseían algún tipo de certificación en el manejo de proyectos, y auscultar si existía diferencia en cuanto a la percepción de importancia de las destrezas dependiendo de la experiencia del gerente de proyecto y si el gerente posee alguna certificación en el manejo de proyectos.

A base de los objetivos de la investigación se desarrollaron cuatro hipótesis de trabajo:
$\mathrm{H}^{1} \quad$ El modelo de destrezas en el manejo de proyectos de índole estratégicos (Rodríguez, 2005) tiene aplicabilidad en el manejo de proyectos estratégicos en la industria farmacéutica en Puerto Rico.

$\mathrm{H}^{2} \quad$ No existen diferencias entre las destrezas de importancia en el manejo de proyectos identificadas en el modelo de destrezas en el manejo de proyectos de índole estratégicos (Rodríguez, 2005) y los resultados de esta investigación.

$\mathrm{H}^{3} \quad$ No existen diferencias en la percepción de importancias de las destrezas entre los gerentes de proyecto con 0-4 años de experiencia y los que poseen 15 años o más de experiencia.

$\mathrm{H}^{4} \quad$ No existen diferencias en la percepción de importancias de las destrezas entre los gerentes que poseen alguna certificación en el manejo de proyectos y los que no poseen certificaciones en el manejo de proyectos.

Las hipótesis fueron puestas a prueba por medio de un cuestionario basado en el modelo de destrezas en el manejo de proyectos de índole estratégicos (Rodríguez, 2005), recopilando información de participantes a través de cuestionarios que cumplan con el perfil profesional de interés en el evento Interphex Puerto Rico 2009, el cual reúne una gran cantidad de profesionales que laboran en la industria farmacéutica. La investigación tuvo un enfoque cuantitativo y descriptivo, con una muestra de 131 cuestionarios, apoyándose en el análisis estadístico para comprobar las hipótesis establecidas. 
El cuestionario consistió en tres partes y se basó en el modelo de destrezas en el manejo de proyectos de índole estratégicos (Rodríguez, 2005). Las primeras dos partes abarcaban preguntas relacionadas con las destrezas técnicas y gerenciales, utilizando una escala Likert de cinco puntos: no importante (1), poco importante (2), algo importante (3), importante (4) y muy importante (5). La tercera parte del cuestionario consistió en nueve preguntas de índole demográficas con una escala nominal. El cuestionario se validó por medio de una muestra representativa de 30 gerentes de proyecto, obteniendo una alfa de Cronbach de 0.894 , lo cual comprobó la validez del instrumento, debido a que un valor mayor o igual de 0.7 es considerado como aceptable (UCLA, 2009).

El perfil de la muestra representativa estudiada fue: mayores de edad y que se desempeñaran como gerente de proyectos de ingeniería o construcción, fueran ejecutivos o personal de apoyo a proyectos, tuvieran experiencia de trabajo en la industria farmacéutica en Puerto Rico y asistentes a Interphex Puerto Rico 2009. La población del estudio fue estimada por medio de la asistencia de personal con el perfil deseado al evento en el 2008. Con un total de 195 participantes con dicho perfil en el evento del 2008, se estableció como meta el obtener 130 cuestionarios para alcanzar un nivel de confianza de $95 \%$ y un intervalo de confianza de 5\% (Rea y Parker, 1997). En el evento se obtuvieron un total de 146 cuestionarios, de los cuales se identificaron un total de 131 cuestionarios válidos, lo cual cumplió con las expectativas del diseño de la muestra.

La variable dependiente del estudio fue la habilidad del gerente en manejar un proyecto de índole estratégico de una forma efectiva, logrando las metas establecidas.
Las destrezas del gerente de proyectos son consideradas las variables independientes (o indicadores) que influencian en su buen desempeño. Estas se dividen en destrezas técnicas (determinar los objetivos del proyecto, determinar los resultados tangibles, habilidad técnica, comunicación del estado del proyecto, desarrollo de itinerario de trabajo, medición del rendimiento del proyecto, respuesta a riesgo, implementación de control de cambios, gerencia del programa de proyectos, gerencia del portafolio de proyectos y gerencia estratégica) y gerenciales (liderazgo del proyecto, flexibilidad, honradez, estilo de comunicación, establecimiento y manejo de expectativas, destrezas organizacionales, solución de problemas y trabajo en equipo).

\section{Resultados}

\section{Resultados demográficos}

De los resultados obtenidos se desprende el perfil del gerente de proyectos de la industria farmacéutica en Puerto Rico: Posee sobre 5 años de experiencia en la gerencia de proyectos (87\%); su título refleja el área de gerencia de proyectos (34\%); maneja proyectos de sobre \$1 millón $(65 \%)$; posee al menos un bachillerato $(62 \%)$; no posee una certificación en manejo de proyectos (78\%); su mayor área de experiencia lo es la industria farmacéutica (89\%); no posee experiencia global $(70 \%)$ y no están certificado como "Project Management Profesional”, PMP (94\%).

Resultados de destrezas técnicas y gerenciales

Debido a que los valores de 4 (importante) y 5 (muy importante) son representativos de una alta percepción de importancia, se decidió utilizar el valor de 4 como el mínimo para identificar una 
destreza como importante en el estudio, lo cual coincide con el criterio utilizado en el modelo de destrezas en el manejo de proyectos de índole estratégicos (Rodríguez, 2005). De los resultados de las destrezas técnicas y gerenciales se desprende que todas obtuvieron una media y una mediana igual o mayor a 4.0, por lo que todas son consideradas como significantes para el estudio. Los resultados obtenidos no exhibieron características de distribuciones normales, por lo cual los análisis fueron realizados utilizando las medianas para cada variable y estadística no paramétrica. indica que las 19 destrezas, 11 de tipo técnico y 8 de tipo gerencial, fueron significativas. Esto coincide con las 19 destrezas identificadas por el modelo de destrezas en el manejo de proyectos de índole estratégicos (Rodríguez, 2005). Basado en los resultados, se acepta la hipótesis nula $\left(\mathrm{H}^{1}\right)$ : el modelo desarrollado por Rodríguez (2005) de destrezas en el manejo de proyectos estratégicos tiene aplicabilidad en la industria farmacéutica en Puerto Rico. Una representación gráfica del modelo de Rodríguez (2005) se muestra en la Figura 3.


Figura 3. Ilustración gráfica del modelo de destrezas en el manejo de proyectos de índole estratégicos (Rodríguez, 2005).

\section{Primera hipótesis}

Para retar la primera hipótesis nula, $\mathrm{H}^{1}$, se procedió a realizar una prueba de estadística descriptiva mediante la comparación de la importancia de las destrezas en el estudio versus las identificadas en el modelo de destrezas en el manejo de proyectos de índole estratégicos (Rodríguez, 2005). Debido a que todas las destrezas obtuvieron una mediana mayor a 4.0, todas son consideradas como importantes para el estudio, lo cual nos

\section{Segunda hipótesis}

Para retar la segunda hipótesis nula, $\mathrm{H}^{2}$, se realizó una prueba de estadística descriptiva, observando la media y el orden de los resultados obtenidos por Rodríguez (2005) y comparándolos con los resultados obtenidos en esta investigación. La tabla 1 presenta un resumen de los resultados obtenidos por Rodríguez (2005). 
Tabla 1

Resumen de los resultados de Rodríguez. (2005) en destrezas en manejo de proyectos

\begin{tabular}{ll}
\hline Destreza & Media \\
\hline Honradez & 4.52 \\
Elaboración itinerario de trabajo & 4.27 \\
Determinar las metas proyecto & 4.24 \\
Determinar los resultados tangibles & 4.19 \\
Comunicación del estado del proyecto & 4.19 \\
Medición del rendimiento del proyecto & 4.11 \\
Estilo de comunicación & 4.10 \\
Liderazgo del proyecto & 4.08 \\
Solución de problemas & 4.02 \\
Gerencia estratégica & 4.02 \\
Trabajo en equipo & 3.99 \\
Respuesta a Riesgo & 3.97 \\
Destrezas organizacionales & 3.94 \\
Establecimiento y manejo de expectativas & 3.90 \\
Flexibilidad & 3.89 \\
Gerencia del programa de proyectos & 3.78 \\
Implementación de control de cambios & 3.76 \\
Habilidad Técnica & 3.69 \\
Gerencia del portafolio de proyectos & 3.69 \\
\hline & \\
\hline
\end{tabular}

De acuerdo al criterio establecido por Rodríguez (2005), las destrezas de importancia en el manejo de proyectos son (en orden de importancia): honradez, elaboración itinerario de trabajo, determinación de las metas proyecto, determinación de los resultados tangibles, comunicación del estado del proyecto, medición del rendimiento del proyecto, estilo de comunicación, liderazgo del proyecto, solución de problemas y gerencia estratégica. Debido a que los resultados disponibles de Rodríguez (2005) no establecen los valores de las medianas de sus variables, no se puede realizar una comparación directa con los resultados del esta investigación. En la tabla 2, se presenta una comparación entre el orden y las destrezas de importancia que se desprenden de ambos estudios.

Tabla 2

Comparación de destrezas de importancia según Rodríguez (2005) con esta investigación

\begin{tabular}{|c|c|c|}
\hline \multirow[b]{2}{*}{ Destreza } & \multicolumn{2}{|c|}{ Comparacion de destrezas } \\
\hline & $\begin{array}{l}\text { Mediana esta } \\
\text { investigación }\end{array}$ & $\begin{array}{l}\text { Media investigación } \\
\text { Rodríguez (2005) }\end{array}$ \\
\hline Determinar las metas del proyecto & 5 & 4.27 \\
\hline Liderazgo del proyecto & 5 & 4.08 \\
\hline Honradez & 5 & 4.52 \\
\hline Determinar los resultados tangibles & 5 & 4.19 \\
\hline Trabajo en equipo & 5 & 3.99 \\
\hline Comunicación del estado del proyecto & 5 & 4.19 \\
\hline Medición del rendimiento del proyecto & 5 & 4.11 \\
\hline Solución de problemas & 4 & 4.02 \\
\hline Respuesta a riesgo & 5 & 3.97 \\
\hline Estilo de comunicación & 5 & 4.10 \\
\hline Flexibilidad & 5 & 3.89 \\
\hline Elaboración del itinerario de trabajo & 5 & 4.27 \\
\hline Establecimiento y manejo de expectativas & 5 & 3.90 \\
\hline Gerencia del programa de proyectos & 4 & 3.78 \\
\hline Gerencia estratégica & 4 & 4.27 \\
\hline Destrezas organizacionales & 4 & 3.94 \\
\hline
\end{tabular}

Sin embargo, de los resultados se desprende que existen diferencias en la significancia de las destrezas. De un total de 19 destrezas estudiadas, tan solo 10 de ellas eran significativas en el modelo de destrezas en el manejo de proyectos de índole estratégicos (Rodríguez, 2005), mientras que todas las destrezas en esta investigación resultaron ser significativas. Esto implica que existen diferencias entre las destrezas de importancia en el manejo de proyectos identificadas en el modelo de Rodríguez (2005), el cual se realizó en los Estados Unidos entre miembros de PMI, y los resultados de esta investigación, 
efectuada con los asistentes al evento Interphex Puerto Rico 2009.

\section{Tercera hipótesis}

Para retar la tercera hipótesis nula $\mathrm{H}^{3}$, se procedió a realizar una prueba de estadística descriptiva mediante la comparación de los resultados de las destrezas de importancia según los gerentes de proyecto que reportaron tener de 0 a 4 años de experiencia versus los que reportaron tener 15 años o más de experiencia. En la tabla 3, se presenta una comparación entre los resultados de los grupos de 0-4 años de experiencia con el grupo de 15 años o más de experiencia.

Tabla 3

Comparación de destrezas de importancia entre participantes de los renglones de 0-4 años y 15 años o más

\begin{tabular}{|c|c|c|}
\hline \multirow[b]{2}{*}{ Destreza } & \multicolumn{2}{|c|}{ Mediana de destrezas } \\
\hline & $\begin{array}{l}0-4 \text { años } \\
\text { experiencia }\end{array}$ & $\begin{array}{c}15 \text { años o } \\
\text { más de experiencia }\end{array}$ \\
\hline $\begin{array}{l}\text { Trabajo en equipo } \\
\text { Medición del rendimiento del }\end{array}$ & 5 & 4 \\
\hline Proyecto & 5 & 5 \\
\hline $\begin{array}{l}\text { Liderazgo del proyecto } \\
\text { Determinar las metas del }\end{array}$ & 5 & 5 \\
\hline Proyecto & 5 & 5 \\
\hline Determinar los resultados Tangibles & 5 & 5 \\
\hline Honradez & 5 & 5 \\
\hline Respuesta a riesgo & 5 & 5 \\
\hline Gerencia del programa de Proyectos & 5 & 5 \\
\hline Elaboración del itinerario de Trabajo & 5 & 5 \\
\hline Solución de problemas & 5 & 5 \\
\hline Flexibilidad & 5 & 5 \\
\hline Comunicación del estado del Proyecto & 5 & 5 \\
\hline Estilo de comunicación & 5 & 5 \\
\hline $\begin{array}{l}\text { Destrezas organizacionales } \\
\text { Establecimiento y manejo de }\end{array}$ & 5 & 5 \\
\hline $\begin{array}{l}\text { Expectativas } \\
\text { Implementación de control de }\end{array}$ & 5 & 5 \\
\hline Cambios & 4 & 4 \\
\hline Gerencia estratégica & 4 & 4 \\
\hline Gerencia del portafolio de Proyectos & 4 & 4 \\
\hline Habilidad técnica & 4 & 4 \\
\hline
\end{tabular}

Al examinar los resultados, no se rechaza la hipótesis nula $\mathrm{H}^{3}$, ya que el valor $\mathrm{Z}$ de 0.38 no sobrepasa el valor crítico de 1.96. Por lo tanto, no existen diferencias en la percepción de importancias de las destrezas entre los gerentes de proyecto con 0-4 años de experiencia y los que poseen 15 años o más de experiencia.

\section{Cuarta hipótesis}

Para retar la cuarta hipótesis nula $\mathrm{H}^{4}$, se procedió a realizar una prueba de estadística descriptiva mediante la comparación de los resultados de las destrezas de importancia según los gerentes de proyecto que reportaron poseer algún tipo de certificación versus los que no poseen certificación alguna. En la tabla 4, se presenta una comparación entre los resultados de los grupos con alguna certificación en proyectos $\mathrm{y}$ sin certificaciones en proyecto.

Tabla 4

Comparación de destrezas de importancia entre participantes de los renglones con una o más certificaciones en gerencia de proyectos y sin certificaciones

\begin{tabular}{lll}
\hline \multicolumn{1}{c}{ Destreza } & Medrana de destrezas \\
& $0-4$ años experiencia & 15 años o \\
& & más de experiencia \\
\hline Trabajo en equipo & 5 & 4 \\
Medición del rendimiento del proyecto & 5 & 5 \\
Liderazgo del proyecto & 5 & 5 \\
Determinar las metas del proyecto & 5 & 5 \\
Determinar los resultados tangibles & 5 & 5 \\
Honradez & 5 & 5 \\
Respuesta a riesgo & 5 & 5 \\
Gerencia del programa de proyectos & 5 & 5 \\
Elaboración del itinerario de trabajo & 5 & 5 \\
Solución de problemas & 4 & 5 \\
Flexibilidad & 5 & 5 \\
Comunicación del estado del proyecto & 4 & 5 \\
Estilo de comunicación & 5 & 5 \\
Destrezas organizacionales & 5 & 5 \\
Establecimiento y manejo de expectativas & 5 & 5 \\
Implementación de control de cambios & 5 & 5 \\
Gerencia estratégica & 5 & 5 \\
Gerencia del portafolio de proyectos & 5 & 5 \\
Habilidad técnica & 5 & 5 \\
\hline & 5 & 5 \\
\hline
\end{tabular}


Al examinar los resultados, no se rechaza la hipótesis nula $\mathrm{H}^{4}$, ya que el valor $\mathrm{Z}$ de 0.49 no sobrepasa el valor crítico de 1.96. Por lo tanto, no existen diferencias en la percepción de importancias de las destrezas entre los gerentes que poseen alguna certificación en el manejo de proyectos y los que no poseen certificaciones en el manejo de proyectos.

\section{Análisis de los resultados y conclusiones}

Esta investigación contribuye a identificar las destrezas de importancia en el manejo de proyectos, específicamente en las industrias farmacéuticas en Puerto Rico. Los datos generados a través del cuestionario resultaron adecuados para la investigación y permitió evaluar cada una de las hipótesis planteadas. Los resultados de la investigación ilustran el perfil de un gerente de proyectos en la industria farmacéutica en Puerto Rico: educado a nivel universitario, con varios años de experiencia, pero poca educación formal en el campo de la gerencia de proyectos. Debido a la complejidad de los proyectos que son desarrollados en esta industria y a la poca tolerancia de la gerencia en aceptar retrasos en proyectos, debido a su efecto negativo en la competencia por lanzar productos nuevos al mercado antes que sus competidores, los gerentes de proyectos necesitan que sus proyectos tengan una ejecución de excelencia.

Una empresa puede obtener el personal adiestrado en gerencia de proyectos tanto acudiendo al mercado laboral como adiestrando al personal de su empresa. Ambas metodologías aumentan la absorción de conocimiento de la organización, de acuerdo a lo postulado por Cohen y Levinthal (1990), mejorando su competitividad, pero la efectividad de una $u$ otra metodología dependerá de la cultura de la empresa. El reclutar un nuevo empleado adiestrado en gerencia de proyectos tiene como ventaja la introducción de "sangre nueva" en la organización, lo cual promueve la introducción de nuevas ideas, pero la desventaja es que él mismo desconoce las políticas e idiosincrasias internas de la misma, lo cual crea una necesidad de adiestramiento interno que pudiera ser larga $y$ compleja. La complejidad pudiera estar asociada a que el conocimiento de la idiosincrasia de la empresa muy bien pudiera ser difícil de enseñar, por ser de tipo tácito (Polanyi, 1966), muy ligado a las acciones, compromiso y envolvimiento en el entorno del trabajo (Nonaka, 1994), necesitando entonces una curva de aprendizaje no deseada. Pudiera resultar más conveniente y práctico el adiestrar al personal interno, conocedor ya de la cultura organizacional. Opciones en programas universitarios disponibles actualmente lo serían, por ejemplo, la especialidad en gerencia en el programa graduado en Administración de Empresas de la Universidad del Turabo y la especialidad en gerencia de construcción del programa graduado en Gerencia Gerencial de la Universidad Politécnica de Puerto Rico. También serían opciones adiestramientos en gerencia de proyectos, tales como los ofrecidos por el Colegio de Ingenieros y Agrimensores de Puerto Rico, la Universidad Politécnica de Puerto Rico o la certificación como PMP de PMI. Cualesquiera de estos adiestramientos les permitiría a sus participantes el adquirir las 
destrezas necesarias en gerencia de proyectos $\mathrm{y}$, poseer todos los gerentes de proyecto de una empresa el mismo nivel de entendimiento en la materia; esto fomentaría un lenguaje común, lo cual es sumamente importante para lograr la cohesión de sus miembros (Cohen y Levinthal, 1990). Esto a su vez fomentaría el sentido de pertenencia y progreso del empleado cuando es adiestrado y/o promovido versus el reclutamiento externo. importancia en el manejo de proyectos, específicamente en la industria farmacéutica en Puerto Rico. Los resultados obtenidos les proveen valiosa información, tanto a los gerentes de proyectos como a los que desean incursionar en este campo, como a la academia, los proveedores de servicios de adiestramiento y las empresas. Para los gerentes de proyecto o aspirantes a serlo, este estudio les indica cuáles son las destrezas que deben poseer para realizar efectivamente sus labores como gerentes de


dependiendo del medio ambiente y de la industria estudiada. En lo relacionado a los años de experiencia (0 a 4 años versus 15 años o más), los resultados indican que no existe una diferencia estadística entre la

Los resultados le proveen a la academia y los proveedores de servicio información de cual debe de ser el enfoque de sus cursos y/o adiestramientos para adiestrar efectivamente a los gerentes de proyecto. opinión de las destrezas de importancia entre ambos grupos, lo cual tiende a indicar que la importancia de las destrezas técnicas y gerenciales no depende de la experiencia. Lo mismo ocurrió al comparar las destrezas de los gerentes con y sin alguna certificación en gerencia de proyecto; se entiende que dicho resultado se debe a que el proceso de certificación en el área de gerencia de proyectos está primordialmente basado en que el gerente de proyectos sea capaz de aprender una metodología estándar en la elaboración de los proyectos; esto no necesariamente le hará cambiar su percepción por la importancia de las destrezas técnicas y gerenciales.

A pesar que los resultados de este estudio solo se consideran válidos para la muestra de gerentes de proyecto que asistieron al evento de Interphex Puerto Rico 2009 y no necesariamente a la población en general, el mismo contribuye en la investigación de las destrezas de

\section{Puerto Rico.}

Los resultados le proveen a la academia y los proveedores de servicio información de cual debe de ser el enfoque de sus cursos y/o adiestramientos para adiestrar efectivamente a los gerentes de proyecto. Por último, los resultados les proveen a las industrias farmacéuticas información en cuanto a cuáles son las destrezas que sus gerentes de proyecto deben tener para ser efectivos en su desempeño.

Basado en los resultados de este estudio, se recomienda que se repita el mismo en eventos futuros de Interphex Puerto Rico u eventos similares donde exista una participación mayor de gerentes de proyectos, tales como las convenciones del Colegio de Ingenieros y Agrimensores de Puerto Rico (CIAPR) y del capítulo de Puerto Rico de PMI. Dichos resultados pudieran ser utilizados para validar o refutar los resultados de esta investigación. Además, se sugiere replicar el estudio en otras industrias en donde laboran los gerentes de proyecto en Puerto Rico, tales como la banca, las firmas de ingeniería y diseño, proyectos del gobierno, etc. Esto podría conducir al desarrollo de un modelo 
de destrezas en el manejo de proyectos para toda industria en Puerto Rico.

\section{Referencias}

Alameda, J. (2006). Las farmacéuticas y la eliminación de la Sección 936. Recuperado de http://www.josealameda.com/publicaciones.htm

Ausburg College (2007). Introduction to Project Management, Recuperado de http://www.augsburg.edu/ppages/ schwalbe/C533 $\underline{2 \_01 \text { FP.pdf }}$

Cleland, D. I. \& King, W. R. (1983). Project Management Handbook. New Jersey: Wiley \& Sons Hoboken.

Cohen, Wesley, M. \& Levinthal, Daniel A. (1990), Absorptive Capacity: A New Perspective on Learning and Innovation, $A S Q, 35$. Recuperado de http://www.informationr.net/ir/182/paper577.html\#.UmsYruLZgt

Enchautegui, R., Rodríguez, A., Segarra, E. (2005), Estudio de Destrezas y Ocupaciones en Mayor Demanda. Estado Libre Asociado de Puerto Rico, Departamento del Trabajo y Recursos Humanos. Recuperado de http://www.netempleopr.org/almis23/PDF/CEDOE/Informe\%20 De\%20Destrezas\%20PARTE\%201\%20\%20En\%20Mayor\%20Demanda.pdf

Nonaka, I. (1994). A dynamic theory of organizational knowledge creation. Organization Science, 5(1), 14-37.

Polanyi, M. (1966). The tacit dimension. London: Routledge and Kegan Paul.

PRIDCO (2006). Recuperado de http://www.eclac.org/publicaciones/xml/3/20443/ L646-4.pdf (accedido en enero 20, 2009).

Project Management Institute (2004). OPM3 Organizational Project Management Maturity Model: Knowledge Foundation. Newton Square, PA.: Project Management Institute.

Rodríguez, A. (2005). Critical factors in hiring, promoting, and designing job descriptions for strategic project managers. Ph.D. thesis, Capella University.
UCLA Academic Technology Services (2009). "What does Cronbach's alpha mean?,Recuperado de

http://www.ats.ucla.edu/stat/spss/faq/alpha.html

\section{Información del autor de correspondencia}

Francisco Quiles Torres

Francisco.quiles@upr.edu

Gerente Senior de Proyectos, Amgen Manufacturing Limited. Escuela de Derecho, UPR, JD (2013); Universidad del Turabo, DBA (2009); Universidad Politécnica de Puerto Rico, MEM (1994); UPR, Recinto Universitario de Mayagüez, BSEE \& MEEE (1990, 2000). 